EPiC Series in Computing
Volume 49, 2017, Pages 33-49
$\begin{aligned} & \text { PROOFS 2017. 6th International Workshop } \\ & \text { on Security Proofs for Embedded Systems }\end{aligned}$

\title{
Automatic Generation of HCCA Resistant Scalar Multiplication Algorithm by Proper Sequencing of Field Multiplier Operands*
}

\author{
Poulami Das, Debapriya Basu Roy, and Debdeep Mukhopadhyay \\ Indian Institute of Technology, Kharagpur, India \\ \{poulami, debapriya, debdeep\}@cse.iitkgp. ernet.in
}

\begin{abstract}
Horizontal collision correlation analysis (HCCA) imposes a serious threat to simple power analysis resistant elliptic curve cryptosystems involving unified algorithms, for e.g. Edward curve unified formula. This attack can be mounted even in presence of differential power analysis resistant randomization schemes. In this paper we have designed an effective countermeasure for HCCA protection, where the dependency of side-channel leakage from a school-book multiplication with the underling multiplier operands is investigated. We have shown how changing the sequence in which the operands are passed to the multiplication algorithm introduces dissimilarity in the information leakage. This disparity has been utilized in constructing a zero-cost countermeasure against HCCA. This countermeasure has been shown to help in HCCA resistivity. Additionally we provide experimental validation for our proposed countermeasure technique on a SASEBO platform. To the best of our knowledge, this is the first time that asymmetry in information leakage has been utilized in designing a side channel countermeasure and successfully applied in an ECC-based crypto-module.
\end{abstract}

\section{Introduction}

Elliptic curve cryptosystems are emerging as a primary choice for securing light-weight embedded devices as it incorporate more security per key bit compared to RSA [18], thus qualifying as a less resource hungry alternative. Also with the recent explosion of internet of things (IoT), applications using light-weight hardware devices are increasing exponentially which in turn make the security of the underlying devices imperative. However, the hardware implementations of cryptographic applications suffer an inevitable insecurity in terms of side-channel leakage, even though the system is theoretically protected. Side channel leakage of information through power consumption [26], electromagnetic (EM) dissipation, acoustic channel [16], etc makes the system weakly protected and may lead to complete secret key recovery. A naíve implementation

*The authors would like to thank ISEA Funding for Research on Next Generation Network Security for partially supporting their work.

U. Kühne, J.-L. Danger and S. Guilley (eds.), PROOFS 2017 (EPiC Series in Computing, vol. 49), pp. 33-49 
of an elliptic curve (EC) scalar multiplication algorithm, consisting of sequential doubling and addition operations, can be broken through simple power analysis (SPA) [11] with only a single trace of execution. This motivates researchers to construct cryptosystems which are inherently secure against SPA. Atomic scheme algorithms have been introduced in [9], [27] which transform the doubling and addition operation into a uniform structure, such that it becomes infeasible to distinguish an addition operation from a doubling from a single power trace. However, these atomic scheme algorithms still involve different formulae for addition and doubling, which has motivated researchers for further unification. In [8] a unified addition formula is designed for a Weierstrass form of elliptic curve, for both addition and doubling. While in [13] a new form of curve, named Edwards curve has been built involving a complete addition formula which gives a valid elliptic curve point as output for any two curve points taken as input, thus taking care of both addition and doubling. Recent extensive research involving use of Edwards curve in cryptosystems reveals its implementation friendliness [4], [5], [25], [20]. Also it is being considered as a safe curve with respect to a number of important factors (ladder security, twist security). [7] contain details on the defined safe curve criteria. Indeed because of the presence of single formula for both point addition and point doubling, an Edwards curve implementation, similarly Brier-Joye unified formula [8] is SPA resistant. We note here that there exists advanced attacks such as differential power analysis (DPA) attack [11] which can exploit a SPA-resistant implementation, thus considered as a serious threat to elliptic curve cryptography (ECC) designs. However, it requires access to a significantly large number of power or EM traces of EC scalar multiplication executions, with a fixed secret key, hence this scenario is not directly applicable to ECDSA, where a secret scalar is used only once. However, the Big Mac attack by [30] introduces an advanced form of single trace attacks later termed as horizontal attacks which exposes even an SPA protected implementation. Several horizontal attack approaches followed the Big Mac analysis in [15, 19, 2] which were mainly focused on RSA based exponentiation algorithms. Authors in [3] have put forward the idea of horizontal attack in case of elliptic curve cryptography. The attack combines methodologies from the well established horizontal attack [30] and the idea of collision attack (introduced in [28]), hence termed as horizontal collision correlation analysis (HCCA) which breaks an atomic scheme ECC algorithm or a unified ECC algorithm equipped with SPA-resistance. Even when the design is protected against advanced attacks such as DPA, refined power analysis [17], address-bit differential attack [21] with effective randomization schemes suggested in [22], [14], HCCA can be launched, thus introducing genuine vulnerability in the implementation. It exploits the relation of the secret key value with a property pertaining to the underlying field multiplications involved in a point doubling and point addition operation. It is a unique property based on the sharing of operands between two field multiplications which holds irrespective of any randomization used at each iteration of the scalar multiplication.

Our contribution Our main contribution in this paper is to show how we can design a zerocost yet effective countermeasure that helps in resisting HCCA. Our contribution in this paper can be summarized as follows

- We coin a term order of operands to define the sequence in which two operands are passed as parameters to a long integer multiplication routine. We show how the information leakage from a multiplication varies when the order of operands in a multiplication is changed. We also derive that the relation between side-channel leakage of two multiplications sharing one (two) common operand (s) is dependent on the order of operands passed to the individual multiplications. We used two distinguishers - Pearson Correlation metric, and Euclidean Distance metric for our analysis. However due to the limited space constraint of this paper, we only show results on Pearson Correlation metric only. 
- Based on this observation, we propose a countermeasure, that can be applied to the existing unified algorithms of ECC to defeat HCCA. The countermeasure converts the unified algorithm into a safer form, such that the relation between side-channel leakage of multiplications based on property of operand sharing cannot be exploited. The countermeasure requires determination of the safe sequence through our proposed algorithm. As a result, there is no additional timing and area overhead on the implementation. We show how the implementation integrated with our proposed countermeasure enhances HCCA resistivity.

- Finally we provide extensive results of mounting HCCA on the proposed countermeasure. The results have been validated on SASEBO-GII with Electromagnetic (EM) traces.

\section{Related Work}

Big Mac analysis [30] introduced the idea of applying differential power analysis along the length of a single exponentiation trace of RSA. It shows how the data dependency during the pre-computation phase can be exploited to identify exponent digits involved in a long integer multiplication during an m-ary RSA exponentiation. The vulnerability is shown to increase if the length of the key increases exposing more multiplication traces to compare with. Authors in [1] applies a novel technique of distinguishing multiplication from squaring operations based on the difference in their expected Hamming weight distribution. However, it is a vertical attack gathering information from several traces along the same region of a long integer multiplication. In [10] the idea of horizontal attack on an RSA exponentiation has been strengthened by exploiting a significant number of potential collision pairs obtained within a long integer multiplication, if the underlying operation is a squaring operation. Multiplication operations are expected to result in less collisions compared to squaring due to the presence of different input operands. In [15] a practical vulnerability of using scalar blinding as a DPA countermeasure has been demonstrated. Due to the sparse form of NIST prime, a portion of the secret key remains unblinded and gets exposed to vertical collision analysis, the rest part of the key is recovered using horizontal attack techniques. In [2] a generic approach is introduced to break an ECC implementation with the help of one template trace per scalar bit. In [19] the vulnerability of regularized algorithms such as Montgomary Ladder [24], Joye's Add-Only scalar multiplication [23] is highlighted, based on collisions of intermediate results obtained from consecutive iterations. In later section we demonstrate the resistance of our countermeasure from the above mentioned horizontal attacks.

\section{Preliminaries}

We discuss the idea of Horizontal Collision Correlation Analysis (HCCA) attack in this section.

\subsection{Horizontal Collision Correlation Analysis}

First we proceed to explain the HCCA attack methodology with the help of an illustration, followed by a summarization of the attack. Before moving to the example describing HCCA, a closer look is given to the field operations underlying ECC doubling and addition operations. It is evident that, ECC point addition and point doubling operations are associated with a number of field multiplication and field addition operations. The underlying field multiplications play an important role in HCCA. The attack is based on the assumption: The adversary can detect when two field multiplications have at least one operand in common [3]. Without loss of generality we 


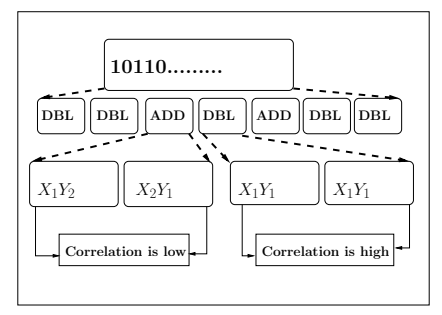

Figure 1: Horizontal Collision Correlation Analysis (HCCA)

consider distinct field elements as $A, B, C, D$ to be used as operands to field multiplications. Then the possible field multiplication pairs will take one of the following forms: 1) $A \times B, C \times D$ sharing no common operand, 2) $A \times B, C \times B$ sharing one common operand, 3) $A \times B, A \times B$ where both the operands are same. Note that here no particular assumptions is made on the order in which the operands are passed. However, operands which are common are generally passed in the same order in the two concerned multiplications. Based on the above class of multiplication pairs, we define the following properties of field multiplication pairs:

- property 1: when a pair of multiplications $\left(m_{i}, m_{j}\right)$ share one (two) common operand (s) among themselves.

- property 1a: when a pair of multiplications $\left(m_{i}, m_{j}\right)$ share exactly one common operand among themselves. For example, the pair $(A \times B, C \times B)$ satisfies property $1 a$.

- property 1b: when a pair of multiplications $\left(m_{i}, m_{j}\right)$ share exactly two operands, i.e. they denote the same multiplications. For example, the pair $(A \times B, A \times B)$ satisfies property $1 b$.

- property 2: when a pair of multiplications $\left(m_{i}, m_{j}\right)$ share no common operand among themselves. For example, the pair $(A \times B, C \times D)$ having independent operands satisfies property 2.

Such relation between field multiplication operations is exploited to identify the doubling and addition operations computed during an ECC scalar multiplication, which in turn is directly dependent on the secret key. Hence identification of doubling and addition operations leads to the recovery of the underlying unknown key. Now we proceed to illustrate the attack scenario of HCCA. Without loss of generality, a key sequence has been considered as $10110 \ldots$ which can be expanded as $D B L, D B L, A D D, D B L, A D D, D B L, D B L, \ldots$, where $D B L$ represents a point doubling operation, while $A D D$ denotes a point addition operation as shown in Figure 1. Each of the $A D D / D B L$ operations consist of underlying field additions and field multiplications. For an instance, it can be observed in Figure 1, that there exists a multiplication pair $\left(X_{1} Y_{2}, X_{2} Y_{1}\right)$ within the addition operation, satisfying property 2 of sharing operands. While a pair $\left(X_{1} Y_{1}\right.$, $\left.X_{1} Y_{1}\right)$ can be found in case of doubling satisfying the property $1 b$ of sharing operands. Now, according to [3], if the correlation between the power traces of two concerned multiplication pairs be considered, the multiplication pair $\left(X_{1} Y_{2}, X_{2} Y_{1}\right)$ should give low correlation value, with respect to the correlation value obtained from the multiplication pair $\left(X_{1} Y_{1}, X_{1} Y_{1}\right)$. If significant difference between the correlation values is obtained, then the doubling and addition operations can be successfully identified, leading to the complete secret key recovery. This is how an attacker can launch HCCA. The detailed intermediate steps of the Edwards curve formula, vulnerable to HCCA can be found in Figure 2. 


\section{Our Proposed Countermeasure}

We propose here a zero-cost countermeasure technique which ensures the resistance of a unified ECC algorithm against horizontal collision correlation attack (HCCA). Our proposed countermeasure centers around the concept of reordering of field operands underlying a field multiplication. It involves transforming the ECC point doubling and point addition operations into a secure form, such that even if condition 1 holds, it is not revealed to the adversary. In other words, the information of one of the operations satisfying property 3 is hidden through our implementation. An ECC implementation integrated with our proposed countermeasure becomes more resistant against HCCA. Our countermeasure requires zero overhead of resources in case of the Edwards curve unified formula as well as Brier-Joye unified formula. It is based on an observation that the leakage from the power consumption is dependent on the ordering of operands in a field multiplication. This discrepancy in leakage occurs as the ordering of the operands brings in asymmetry in the leakage, which we exploit to develop our countermeasure. We note that although the concept of asymmetric leakage has been addressed in [29] in case of multipliers and swapping of operands has been suggested as a potential countermeasure, however authors of [29] do not exploit its applicability to any ECC cryptosystem. To the best of our knowledge, this is the first countermeasure design for any elliptic curve cryptosystem which utilizes asymmetry in information leakage of multiplier operands.

\subsection{Asymmetric Leakage of Field Multiplication}

In this section we explain our theoretical rationale behind the asymmetric leakage of field multiplications, which contribute in constructing our countermeasure scheme. We begin our discussion with an introduction to Long Integer Multiplication (LIM) shown in Algorithm 1. The long integer multiplication routine is called to compute underlying field multiplications involved in the ECC point addition, doubling operations. The LIM takes two field operands $X, Y$ as input and outputs their product $X Y$. Each of the field operands passed as parameter in the LIM routine consists of underlying $t$ words, each of size $w$. The result can be of size $2 t$, and is stored in a register of length $2 t$ words. The algorithm is run $\mathcal{O}\left(t^{2}\right)$ times.

To establish the reasoning behind asymmetry in leakage of field multiplications, we introduce here an information leakage model which will guide us towards the theoretical basis of our countermeasure. Generally, in case of an iterative algorithm, a calculation $C_{i}$ is identified, which is operated at each iteration of the algorithm execution. The output $O_{i}$ of the calculation $C_{i}$ is updated at every iteration to a specific register location. The value of the output $O_{i}$ computed and stored at each iteration leaks an information. This information leakage is denoted as $l\left(O_{i}\right)$, which can be approximated using a function of $O_{i}$ i.e $f\left(O_{i}\right)$. The information leakage at each iteration gets augmented iteratively to result in a vector $\left\langle f\left(O_{i}\right)\right\rangle$. In case of Algorithm 1, we consider an instance of the long integer multiplication run with input field operands $A=$ $\left(a_{t}, a_{t-1}, \ldots, a_{2}, a_{1}\right), B=\left(b_{t}, b_{t-1}, \ldots, b_{2}, b_{1}\right)$ which results in the output $A \times B$. At $(i, j)^{t h}$ iteration we can associate the calculation $C_{i, j}$ with the partial product computation $a_{i} \times b_{j}$. The output of the partial product $O_{i, j}=a_{i} b_{j}$ is stored in every iteration, which leaks an information $l\left(O_{i, j}\right)$. We assume that the information leakage $l\left(O_{i, j}\right)$ follows Hamming weight power model. As a result, the function $f\left(O_{i, j}\right)$ is approximated with the help of the Hamming weight of the output value $O_{i, j}$. So we consider $f\left(O_{i, j}\right)=H\left(O_{i, j}\right)$, where $H(x)$ implies the Hamming weight of the value $x$. Based on the leakage model considered, the information leakage of long integer multiplication can be represented by an augmented vector, denoted as $\left\langle H\left(O_{i}\right)\right\rangle$, or $<H\left(a_{i} b_{j}\right)>$. It is evident from Algorithm 1 that the sequence of partial products changes when the order of the operands passed as parameter to the LIM routine is swapped. We consider the 


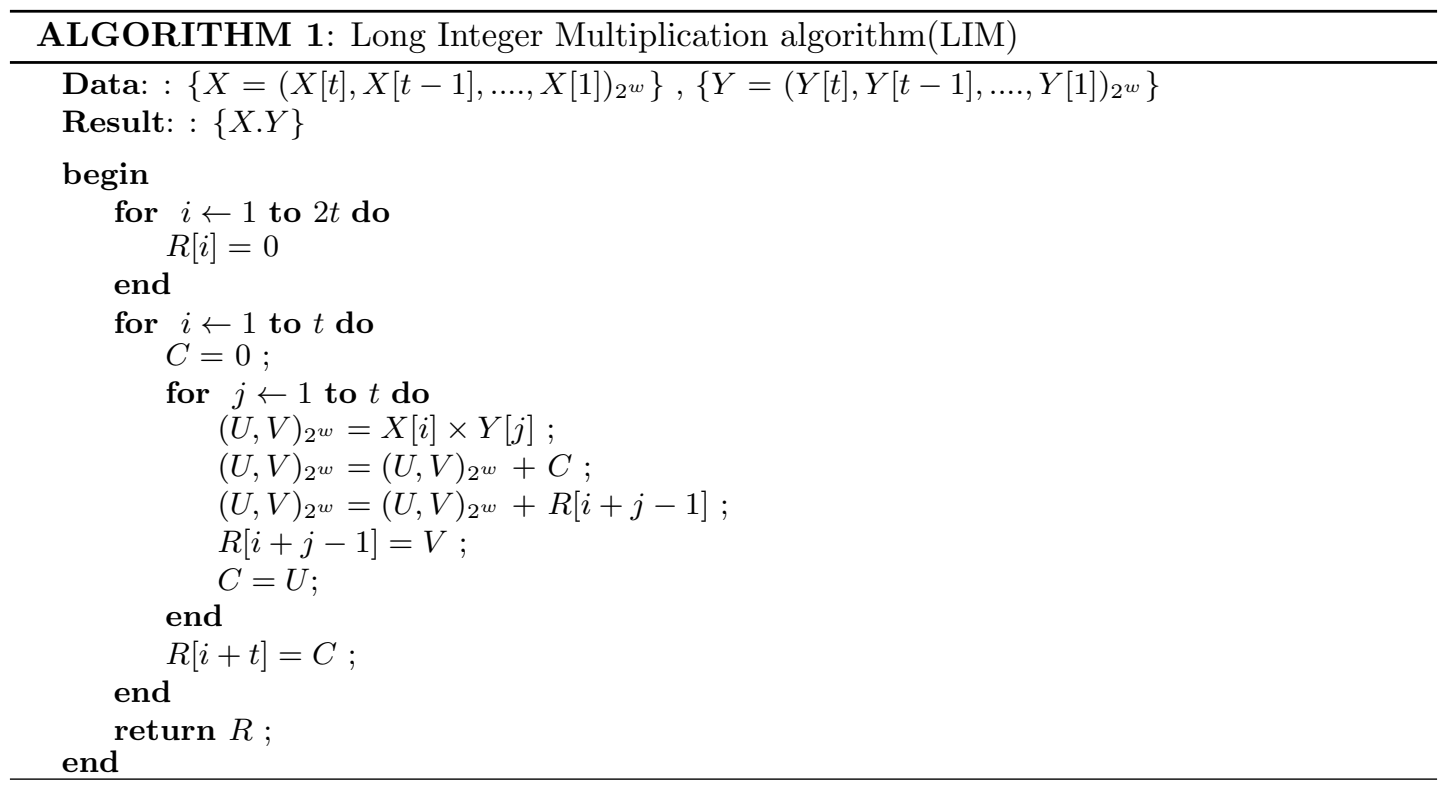

information leakage $l\left(a_{i}, b_{j}\right)$ at each iteration, corresponding to partial product $a_{i} \times b_{j}$ computed during an instance of $\operatorname{LIM}(A, B)$ execution. It is observed that the vector is formed as $<l_{\left(a_{0}, b_{0}\right)}$, $l_{\left(a_{0}, b_{1}\right)}, \ldots, l_{\left(a_{0}, b_{t-1}\right)}, \ldots, l_{\left(a_{t-1}, b_{t-1}\right)}>$. While the one obtained during computation of $\operatorname{LIM}(B$, A) can be presented as $<l_{\left(b_{0}, a_{0}\right)}, l_{\left(b_{0}, a_{1}\right)}, \ldots, l_{\left(b_{0}, a_{t-1}\right)}, \ldots, l_{\left(b_{t-1}, a_{t-1}\right)}>$. This asymmetry in the sequence of the two vectors contribute as a distinguisher between two multiplications.

To calculate the relationship between information leakage of two long integer multiplications, we have considered the following metrics

\subsubsection{Pearson Correlation Metric:}

Considering underlying field operands as: $A, B, A^{\prime}, B^{\prime}$, the correlation between two long integer multiplications $\operatorname{LIM}(A, B)$ and $\operatorname{LIM}\left(A^{\prime}, B^{\prime}\right)$ can be approximated with the Pearson correlation coefficient computed between two vectors $\left\langle H\left(a_{i} b_{j}\right)\right\rangle,\left\langle H\left(a_{i}^{\prime} b_{j}^{\prime}\right)\right\rangle$ (following similar notation as above). Let us denote the two vectors as $H(A B)$ and $H\left(A^{\prime} B^{\prime}\right)$ respectively. The correlation is obtained as follows

$$
\rho=\frac{\text { Covariance }\left(H(A B), H\left(A^{\prime} B^{\prime}\right)\right)}{\sqrt{\text { Variance }(H(A B))} \sqrt{\text { Variance }\left(H\left(A^{\prime} B^{\prime}\right)\right)}}
$$

We now onwards denote the covariance between two vectors as $\operatorname{cov}\left(H(A B), H\left(A^{\prime} B^{\prime}\right)\right)$, variance as $\operatorname{var}(H(A B))$. The standard deviation from the information leakage of a long integer multiplication $\operatorname{LIM}(A, B)$ is denoted as $\operatorname{std}(A B)$. It is obtained as below

$$
\operatorname{std}(A B)=\operatorname{std}(\langle H(A B)\rangle)=\sqrt{\frac{\sum_{i=0, j=0}^{t-1} H\left(a_{i} b_{j}\right)^{2}}{t^{2}}-\left(\frac{\sum_{i=0, j=0}^{t-1} H\left(a_{i} b_{j}\right)}{t^{2}}\right)^{2}}
$$

We define four correlations based on following long integer multiplications $\operatorname{LIM}(A, B)$, $\operatorname{LIM}(B, C), \operatorname{LIM}(C, B), \operatorname{LIM}(C, D)$. The following correlation is obtained from $\operatorname{LIM}(A, B)$ 
and $\operatorname{LIM}(C, B)$

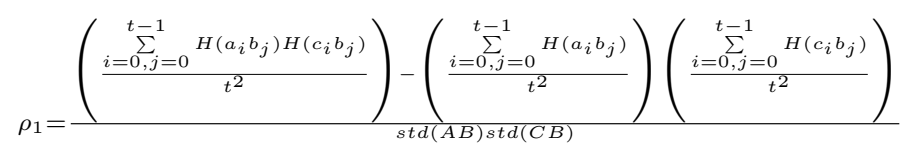

where we denote $\sum_{i=0, j=0}^{t-1} H\left(a_{i} b_{j}\right) H\left(c_{i} b_{j}\right)$ as $\alpha$, where $\alpha$ can be expanded as

$$
\begin{aligned}
\alpha= & \left(H\left(a_{0} b_{0}\right) H\left(c_{0} b_{0}\right)+H\left(a_{0} b_{1}\right) H\left(c_{0} b_{1}\right)+\ldots+H\left(a_{0} b_{t-1}\right)\left(c_{0} b_{t-1}\right)\right. \\
& \left.+H\left(a_{1} b_{0}\right) H\left(c_{1} b_{0}\right)+\ldots+H\left(a_{t-1} b_{t-1}\right) H\left(c_{t-1} b_{t-1}\right)\right)
\end{aligned}
$$

The following correlation is obtained from $\operatorname{LIM}(A, B)$ and $\operatorname{LIM}(B, C)$

$$
\rho_{2}=\frac{\left(\frac{\substack{t-1 \\ i=0, j=0} H\left(a_{i} b_{j}\right) H\left(b_{i} c_{j}\right)}{t^{2}}\right)-\left(\frac{\substack{t-1 \\ i=0, j=0}}{t^{2}}\right)\left(a_{i} b_{j}\right)}{s t d(A B) \operatorname{std}(B C)}
$$

where $\sum_{i=0, j=0}^{t-1} H\left(a_{i} b_{j}\right) H\left(b_{i} c_{j}\right)$ can be expressed as $\beta$, which takes the form

$$
\begin{aligned}
\beta= & \left(H\left(a_{0} b_{0}\right) H\left(b_{0} c_{0}\right)+H\left(a_{0} b_{1}\right) H\left(b_{0} c_{1}\right)+\ldots+H\left(a_{0} b_{t-1}\right) h\left(b_{0} c_{t-1}\right)\right. \\
& \left.+H\left(a_{1} b_{0}\right) h\left(b_{1} c_{0}\right)+\ldots+H\left(a_{t-1} b_{t-1}\right) H\left(b_{t-1} c_{t-1}\right)\right)
\end{aligned}
$$

Here we consider the correlation coefficient between a multiplication pair with property 2 , computed from $\operatorname{LIM}(A, B)$ and $\operatorname{LIM}(C, D)$.

$$
\rho_{3}=\frac{\left(\frac{\substack{t-1 \\ i=0, j=0}}{i=\left(a_{i} b_{j}\right) H\left(c_{i} d_{j}\right)}\right)-\left(\frac{\sum_{i=0, j=0}^{t-1} H\left(a_{i} b_{j}\right)}{t^{2}}\right)\left(\frac{\sum_{i=0, j=0}^{t-1} H\left(c_{i} d_{j}\right)}{t^{2}}\right)}{t^{2}}
$$

where $\sum_{i=0, j=0}^{t-1} H\left(a_{i} b_{j}\right) H\left(c_{i} d_{j}\right)$ is coined as $\gamma$, represented as

$$
\begin{aligned}
\gamma= & \left(H\left(a_{0} b_{0}\right) H\left(c_{0} d_{0}\right)+H\left(a_{0} b_{1}\right) H\left(c_{0} d_{1}\right)+\ldots+H\left(a_{0} b_{t-1}\right) H\left(c_{0} d_{t-1}\right)\right. \\
& \left.+H\left(a_{1} b_{0}\right) H\left(c_{1} d_{0}\right)+\ldots+H\left(a_{t-1} b_{t-1}\right) H\left(c_{t-1} d_{t-1}\right)\right)
\end{aligned}
$$

We develop here few Lemmas which will be required consequently to support the theoretical foundation of our countermeasure. As defined above, $A$ and $B$ denote two field multiplication operands which will be used as parameters in the LIM routine. Now we proceed to the Lemmas.

Lemma 4.1. The standard deviation of a Hamming weight vector obtained from $\operatorname{LIM}(A, B)$ is same as that obtained as $\operatorname{LIM}(B, A)$, i.e $\operatorname{std}(A B)=\operatorname{std}(B A)$.

Proof The vector composed from leakage information of $\operatorname{LIM}(A, B)$ can be expanded as $<H\left(a_{0}, b_{0}\right), H\left(a_{0}, b_{1}\right), \ldots, H\left(a_{0}, b_{t-1}\right), \ldots, H\left(a_{t-1}, b_{t-1}\right)>$. While the vector obtained from leakage information of $\operatorname{LIM}(B, A)$ is represented as $<H\left(b_{0}, a_{0}\right), H\left(b_{0}, a_{1}\right), \ldots, H\left(b_{0}, a_{t-1}\right), \ldots$, $H\left(b_{t-1}, a_{t-1}\right)>$. It can be observed that the two vectors are two different arrangements of same underlying elements. As a result, $\operatorname{std}(A B)=\operatorname{std}(B A)$. Hence proved.

If we denote mean $(X)$ as the mean value of a vector $X$, on the basis of a similar argument we can also show that mean $(A B)=\operatorname{mean}(B A)$.

Lemma 4.2. $\operatorname{cov}(H(A B), H(C B)) \neq \operatorname{cov}(H(A B), H(B C))$. When $C=A, \operatorname{cov}(H(A B), H(A B))$ $\neq \operatorname{cov}(H(A B), H(B A))$. 
Proof The two covariances $\operatorname{cov}(H(A B), H(C B))$ and $\operatorname{cov}(H(A B), H(B C))$, can be represented as

$$
\begin{aligned}
\operatorname{cov}(H(A B), H(C B)) & =\alpha-\text { mean }(A B) \text { mean }(C B) \\
\operatorname{cov}(H(A B), H(B C)) & =\beta-\text { mean }(A B) \text { mean }(B C) \\
& =\beta-\text { mean }(A B) \text { mean }(C B)
\end{aligned}
$$

Since, from Lemma 2. mean $(B C)=$ mean $(C B)$, the second term in both the covariances are mean $(A B)$ mean $(C B)$. Also, from equations 4 and $6, \alpha \neq \beta$, as a result we can conclude

$$
\operatorname{cov}(H(A B), H(C B)) \neq \operatorname{cov}(H(A B), H(B C)) .
$$

When $C=A$ : from equation 4 and 6 , we show that still $\alpha \neq \beta$. The value of $\alpha$ can be expressed as

$$
\begin{aligned}
\alpha= & \left(H\left(a_{0} b_{0}\right) H\left(a_{0} b_{0}\right)+H\left(a_{0} b_{1}\right)\left(a_{0} b_{1}\right)+\ldots+H\left(a_{0} b_{t-1}\right) H\left(a_{0} b_{t-1}\right)\right. \\
& \left.+H\left(a_{1} b_{0}\right) H\left(a_{1} b_{0}\right)+\ldots+H\left(a_{t-1} b_{t-1}\right) H\left(a_{t-1} b_{t-1}\right)\right) \\
= & \left(H\left(a_{0} b_{0}\right)^{2}+H\left(a_{0} b_{1}\right)^{2}+\ldots+H\left(a_{0} b_{t-1}\right)^{2}\right. \\
& \left.+H\left(a_{1} b_{0}\right)^{2}+\ldots+H\left(a_{t-1} b_{t-1}\right)^{2}\right)
\end{aligned}
$$

while $\beta$ can be reduced as

$$
\begin{aligned}
\beta= & \left(H\left(a_{0} b_{0}\right) H\left(b_{0} a_{0}\right)+H\left(a_{0} b_{1}\right) H\left(b_{0} a_{1}\right)+\ldots+H\left(a_{0} b_{t-1}\right) H\left(b_{0} a_{t-1}\right)\right. \\
& \left.+H\left(a_{1} b_{0}\right) H\left(b_{1} a_{0}\right)+\ldots+H\left(a_{t-1} b_{t-1}\right) H\left(b_{t-1} a_{t-1}\right)\right) \\
= & \left(H\left(a_{0} b_{0}\right)^{2}+H\left(a_{0} b_{1}\right)\left(b_{0} a_{1}\right)+\ldots+H\left(a_{0} b_{t-1}\right) H\left(b_{0} a_{t-1}\right)\right. \\
& \left.+H\left(a_{1} b_{0}\right) h\left(b_{1} a_{0}\right)+\ldots+H\left(a_{t-1} b_{t-1}\right)^{2}\right) .
\end{aligned}
$$

From equations 11 and 12 , we can observe that $\alpha \neq \beta$. As a result, when $C=A$, we can conclude similarly that

$$
\operatorname{cov}(H(A B), H(A B)) \neq \operatorname{cov}(H(A B), H(B A)) .
$$

Lemma 4.3. $\rho_{1}>\rho_{2}$ for the case: $A=C$.

Proof When $A=C$, precisely the two multiplications pairs considered are: $(\operatorname{LIM}(A, B)$, $\operatorname{LIM}(A, B))$ and $(\operatorname{LIM}(A, B), \operatorname{LIM}(B, A))$. The correlation $\rho_{1}$ between $(\operatorname{LIM}(A, B), \operatorname{LiM}(A$, $B)$ ) can be computed as

$$
\begin{aligned}
\rho_{1} & =\frac{\operatorname{cov}(H(A B), H(A B))}{\sqrt{\operatorname{var}(H(A B))} \sqrt{\operatorname{var}(H(A B))}} \\
& =\frac{\operatorname{var}(H(A B))}{\operatorname{var}(H(A B))}, \text { since } \operatorname{cov}(X, X)=\operatorname{var}(X) \\
& =1
\end{aligned}
$$

While, the correlation $\rho_{2}$ between $(\operatorname{LIM}(A, B), \operatorname{LIM}(B, A))$ can be computed as

$$
\begin{aligned}
\rho_{2} & =\frac{\operatorname{cov}(H(A B), H(B A))}{\sqrt{\operatorname{var}(H(A B))} \sqrt{\operatorname{var}(H(B A))}} \\
& =\frac{\operatorname{cov}(H(A B), H(B A))}{\operatorname{var}(H(A B))} \\
& <1
\end{aligned}
$$

Since from Lemma 3,

$$
\operatorname{cov}(H(A B), H(A B)) \neq \operatorname{cov}(H(A B), H(B A)) .
$$


Hence it is proved that $\rho_{1}>\rho_{2}$, when $C=A$.

With the help of the lemmas discussed above, we make the following observations:

Observation 1: $\rho_{\mathbf{1}} \neq \rho_{\mathbf{2}}$ From equations 3, 5, we can recollect the mathematical forms of $\rho_{1}$ and $\rho_{2}$. From Lemma 4.1, we can conclude that $\operatorname{std}(A B)=\operatorname{std}(B A)$. As a result, the denominators in case of both the correlations are equal. From Lemma 4.2 we have the result that

$$
\operatorname{Cov}(H(A B), H(C B)) \neq \operatorname{Cov}(H(A B), H(B C)) .
$$

Consequently numerators of the two correlations are unequal. Also, since From Lemma 4.1, mean $(A B)=$ mean $(B A)$, the difference in value arises from the unequal values of $\alpha$ and $\beta$. We give a closer look at the forms of $\alpha$ and $\beta$ to observe that: 1) each term in $\alpha$ takes the form $H\left(a_{i} b_{j}\right) H\left(c_{i} b_{j}\right)$ where the word multiplications share operand $\left.b_{j} .2\right)$ each term in $\beta$ is of the form $H\left(a_{i} b_{j}\right) H\left(b_{i} c_{j}\right)$, where the word multiplications have no common operand. each term of $\alpha$ and $\beta$ take different form yielding different values. As a result, $\rho_{1}$ is clearly not same as $\rho_{2}$.

Observation 2: $\rho_{\mathbf{2}}$ and $\rho_{\mathbf{3}}$ are indistinguishable To make a comparison between the values of $\rho_{2}$ and $\rho_{3}$, we look at the form of each of the terms present in the two equations take: 1) each term in $\beta$ is of the form $H\left(a_{i} b_{j}\right) H\left(b_{i} c_{j}\right)$, where the word multiplications have no common operand. 2) each term in $\gamma$ is of the form $H\left(a_{i} b_{j}\right) H\left(c_{i} d_{j}\right)$, where the word multiplications are devoid of any common term. The two forms $H\left(a_{i} b_{j}\right) H\left(b_{i} c_{j}\right)$ and $H\left(a_{i} b_{j}\right) H\left(c_{i} d_{j}\right)$ are indistinguishable, hence rendering $\beta$ and $\gamma$ being indistinguishable. We conclude from our observation that, the two correlation coefficients take similar form.

Observation 3: $\rho_{1}>\rho_{2}$ for a multiplication pair with property 1b A multiplication pair satisfying property $1 b$, implies same multiplications are being computed. From Lemma 4.3, we obtain that in such a case $\rho_{1}$ will always be greater than $\rho_{2}$ irrespective of the underlying field element values involved. Hence $\rho_{1}>\rho_{2}$ occurs with high probability in such a case.

From the above observations, the importance of ordering of operands in underlying field multiplications can be inferred. Based on our inference, we suggest that the information leakage due to sharing of operands can be hidden by operand reordering. This fact has been exploited in designing our countermeasure which will be explained in the following subsection.

\subsection{Preventing HCCA by choosing safe sequence}

The countermeasure is designed on the basis of the idea of reordering of operands discussed in the previous subsection. It attempts to transform the series of field multiplications underlying ECC point doubling and point addition operation into a HCCA - resistant form. In other words, it makes the implementation secure against HCCA. As can be noted in section 3.1, an ECC implementation becomes vulnerable to HCCA if only one of the addition or doubling operation satisfies property 3 . The idea is to alter the operation containing property 3 , into a form where information regarding operand sharing between field multiplications is hidden. Consequently it is not revealed to the adversary whether any doubling or addition operation contains property 3 or not. Hence the basis of distinction between doubling and addition operation is concealed.

We swap the operands, to blur the correlation between a pair of multiplications sharing operand (s). Let the multiplications be $(A \times B, A \times B)$ computed as $A \times B$ and $B \times A$. $\operatorname{LIM}(A, B)$ gives the expansion $\left(<l_{\left(a_{0}, b_{0}\right)}, l_{\left(a_{0}, b_{1}\right)}, \ldots, l_{\left(a_{0}, b_{t-1}\right)}, \ldots, a_{\left(b_{t-1}, b_{t-1}\right)}>\right), \operatorname{LIM}(B, A)$ leads to $\left(<l_{\left(b_{0}, a_{0}\right)}, l_{\left(b_{0}, a_{1}\right)}, \ldots, l_{\left(b_{0}, a_{t-1}\right)}, \ldots, l_{\left(b_{t-1}, a_{t-1}\right)}>\right)$. Here after swapping of operands, still the first and last partial products namely $a_{0} b_{0}$ and $a_{(t-1)} b_{(t-1)}$ are same for both $A \times B$ and $B \times A$ and hence an attacker may just focus on these two squares for getting similarity 


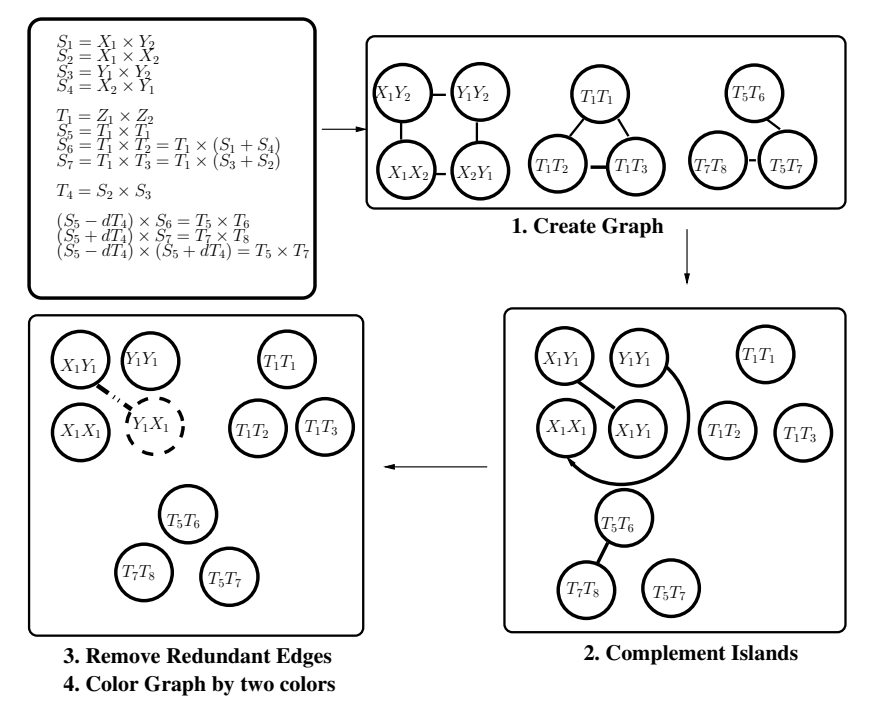

Figure 2: Safe sequence transformation of Edwards curve formula

between $A \times B$ and $B \times A$. However, here we would like to comment that firstly, it would be extremely challenging for an attacker to extract accurately the power (EM) trace corresponding to the partial products within a long integer multiplication, where the long integer multiplication needs to be correctly located within the entire trace. If the partial product is computed as point multiplication within a clock cycle time, the trace corresponding to the partial products will be also small and give insufficient information. Secondly, if the partial products are computed for fairly large base width of 64 bits or above, then it is suggested that they be calculated as long integer multiplications also (64 bits $=4 w$, where $w=16$ bits). Since the partial products are themselves always expanded in the swapping manner $\left(\left(a_{0} b_{0}\right)\right.$ in $A \times B,\left(b_{0} a_{0}\right)$ in $\left.B \times A\right)$, so computing them as long integer multiplications again diffuses the similarity between the partial products.

It should be noted that the transformation technique mainly involves rearrangement of multiplication operands. This process does not incorporate any randomization or any extra operation. Therefore the cost of this countermeasure step is zero in terms of area as well as timing overhead. Moreover, the order of operands are decided beforehand and can be precomputed before implementing the design, requiring only one time effort from the designer's point of view. We design an algorithm, named safe_sequence_converter routine presented in Algorithm 2 which takes care of the transformation process of our countermeasure. We proceed to portray our transformation mechanism through an illustration, which will be followed by a description of our designed Algorithm 2.

We have considered the Edwards curve unified formula shown in [6] for explaining our conversion scheme. It can be noted that the Edwards curve unified formula involves a single formula which is used for both addition and doubling. It underlies a series of field multiplication operations which have been listed in Figure 2. We note that the multiplications are written with respect to the point addition operation, i.e when two distinct points $\left(X_{1}, Y_{1}, Z_{1}\right)$, and $\left(X_{2}, Y_{2}, Z_{2}\right)$ are taken as input. To construct a safe sequence we need to find out which are the multiplications which share operands among themselves. To do so, we construct an undirected graph with the individual multiplications as the graph vertices, whereas an edge is constructed 
between two graph vertices if the two underlying multiplications satisfy property 1 of sharing operands (edge property). We observe in the Figure 2 how edges are formed between $\left(X_{1} X_{2}\right.$, $\left.X_{1} Y_{2}\right),\left(X_{1} X_{2}, X_{2} Y_{1}\right),\left(Y_{1} Y_{2}, X_{1} Y_{2}\right)$ and so on. Furthermore, we witness that the graph is not completely connected, instead it is composed of a number of islands. One may argue that, multiplications such as $T_{5} T_{6}$ involve operand $T_{6}$ which is of the form $T_{1} T_{2}$ (here $T_{1}=Z_{1} Z_{2}$, $T_{2}=S_{1}+S_{4}$ ), so it is sharing a common operand $T_{1}$ (with $T_{1} T_{1}, T_{1} T_{2}$ or $T_{1} T_{3}$ ). This is actually not true because, the multiplication output of $\left(T_{1} T_{2}\right) \bmod F_{p}$, where $F_{p}$ is the underlying field prime, is stored in the location $T_{6}$, and hence it is statistically independent from $T_{1}$. Now we make a crucial observation that, the operand sharing obtained from the graph considered reveals all the operand sharing multiplications which will be present in the addition operation. But if we consider the graph corresponding to the doubling operation where points $\left(X_{1}, Y_{1}, Z_{1}\right)$, and $\left(X_{2}, Y_{2}, Z_{2}\right)$ are the same, it can be observed that the previous operand sharing will still be present along with some possible extra operand sharing vertices. So the operand sharing edges obtained from the addition operation graph illustrated above are the edges common to both addition and doubling operations. As a result, they do not qualify in distinguishing between addition and doubling operations.

Evidently, the operand sharing edges which are found only in case of doubling operation may contribute in the distinction. To get a closer look we consider the complements of the islands of our previously constructed graph. Note that we are not interested in the edges between islands in the complement graph because they do not share operands among themselves. We also replace the vertex values with the respective forms of doubling operation. For example, $X_{1} Y_{2}$ will be replaced with $X_{1} Y_{1}$. The complement of the islands are considered here to concentrate on those edges which will be formed only in case of doubling operation. However, the complement of the islands will include both essential edges (for e.g edge between two vertices each containing value $X_{1} Y_{1}$ ) as well as redundant edges (for e.g. edge between two vertices with values $X_{1} X_{1}$ and $Y_{1} Y_{1}$ respectively which do not satisfy the edge property). We remove the redundant edges, and look only at the essential edges because they are the ones which will help in distinguishing an addition operation from a doubling operation. In this case, doubling operation involves $X_{1} Y_{1}, X_{1} Y_{1}$ operated twice, which are satisfying property $1 b$. On the other hand, addition operation consists of two underlying multiplications $X_{2} Y_{1}, X_{1} Y_{2}$ satisfying property 2 of sharing operands. Thus they successfully depict scenario 1 of HCCA. Based on our observation 2 and observation 3, we rearrange the multiplications as $X_{1} Y_{1}$ and $Y_{1} X_{1}$, so that their operand sharing property remains hidden. Thus, the information leakage for the pair $\operatorname{LIM}\left(X_{1}, Y_{1}\right)$, $\operatorname{LIM}\left(Y_{1}, X_{1}\right)$ will be similar to that of the pair $\operatorname{LIM}\left(X_{2}, Y_{1}\right), \operatorname{LIM}\left(Y_{1}, X_{2}\right)$. (here we refer to the long integer multiplication routine LIM). So we suggest to swap the order of operands of the second multiplication.

From lemma 4.4 stated next we get that the problem of swapping operands of field multiplications can be solved by the problem of two-colorability of a graph. So if the final reduced graph with the islands containing essential edges be two-colorable, then we proceed to color the graph with two colors, and eventually swap the operands of those vertices which belong to the class of one particular color.

In a similar fashion, we transform the Brier-Joye unified formula shown in [8] into a secure structure. The transformation steps corresponding to the Brier-Joye formula is portrayed in Figure 3.

Before proceeding to state the following lemma 4.4, we give here a rationale behind the operand swapping problem formulation. In our operand swapping problem, we need to identify a set of vertices which need to go through operand swapping, keeping other vertices intact as before so that the overall set reaches a secure form. So it is depictable that the vertex set needs 


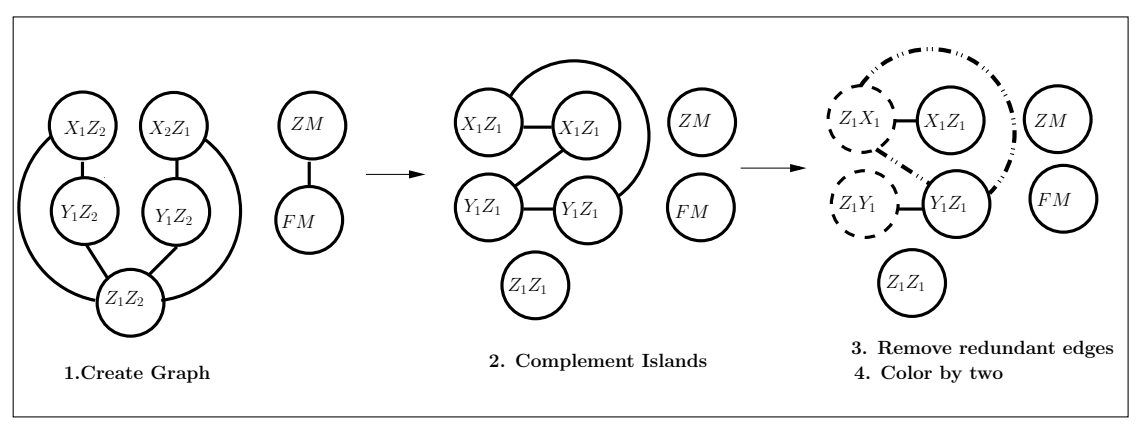

Figure 3: Safe sequence transformation of Brier-Joye unified formula

to be partitioned into two sets. The set of vertices which requires operand swapping is called the swap set, while the other set is named as uninterrupted set. Also it can be perceived that in any edge, since the edge has been created due to operand sharing of two vertices, one of the vertex of the edge should be swapped, thus should belong to swap set. While the other vertex should belong to the uninterrupted set. Furthermore, there does not exist an edge such that both of their end vertices belong to the swap set, or the uninterrupted set. Suppose there exists one such edge, then if both vertices belong to the swap set then it implies in case of both the vertices, the vertex operands have been swapped. But this is equivalent to the state before swapping. For example, it means a vertex pair $\left(X_{1} Y_{1}, X_{1} Y_{1}\right)$ has been swapped to $\left(Y_{1} X_{1}\right.$, $\left.Y_{1} X_{1}\right)$, which does not solve our aim of information masking through operand swapping. This is because the correlation between both the mentioned pairs will be higher with respect to the pair $\left(X_{1} Y_{1}, Y_{1} X_{1}\right)$, as has been proved in lemma 4.3. From this it directly follows why must the vertex ends of any edge belonging to the set $E$ should not belong to the same set (swap set or uninterrupted set). Naturally, it is also understood why the vertices belonging to either swap set or uninterrupted set do not contain any edge between themselves. Now we define the operand swapping problem more formally followed by stating the Two-colorability problem of graph.

Operand swapping problem or problem a: Given an undirected graph $G$ denoted by the set $\{V$, $E\}$, whether there exists a partition of $V$ as $\left(V_{1}, V_{2}\right)$ with following conditions: 1) $V_{1}$ or swap set, consists of elements as $\{v \mid$ operands of v should be swapped $\}$. 2) $V_{2}$ or uninterrupted set, can be presented as $\{v \mid$ operands of v should not be disturbed $\}$. 3) the edge set $E$ is of the form $\left\{e \mid e=\left(v_{i}, v_{j}\right)\right.$, where $\left(v_{i} \in V_{1}, v_{j} \in V_{2}\right)$ or $\left.\left(v_{i} \in V_{2}, v_{j} \in V_{1}\right)\right\}$.

Two-colorability problem of graph or problem $b$ : Given a graph $G$ as set $\{V, E\}$, whether the vertices of the graph can be colored with two colors, such that no two vertices sharing the same edge contain the same color i,e in other words to check whether the graph is a bipartite graph. Now we are ready to state the lemma 4.4.

Lemma 4.4. The problem of swapping of vertex operands (multiplication operands) in an undirected graph is polynomial time reducible to the problem of two-colorability of a graph.

Proof An instance of graph $G$ is fed to the problem $b$, which returns the decision in polynomial time whether the input graph is two-colorable or not. If the answer is yes, then the graph is passed to a graph coloring algorithm that returns the resultant graph colored with two colors. Without loss of generality the two colors can be named as color1 and color2. We define the set of vertices colored with color1 as swap set, while the set of vertices colored with color2 as uninterrupted set. Thus we have determined a solution for the instance of problem a. Hence 
proved.

Now we give a closer look at the correctness of the polynomial reduction of problem a into problem $b$. As was mentioned in the above proof, the solution for the instance of the graph considered corresponding to problem $b$ gives back the graph instance colored with two colors, based on the graph coloring algorithm. The vertices having color1 form set1, while the vertices colored with color2 form a set2. The vertices within set1 do not contain any edge between them, similarly in set2, no two vertices are connected by an edge. For every edge in $E$, two vertices are colored with two distinct colors, which implies the two vertices belong to two different vertex sets. We can consider set1 as the swap set, on the other hand the set2 can be considered as the uninterrupted set required for the solution of problem a. The sets obtained from solution to problem $b$ also satisfies the condition for the edge set that every edge should contain vertices belonging to the two different sets, so that for every edge the vertex belonging to the swap set should undergo operand swapping, while the other vertex from uninterrupted set should remain unaltered. That is why solution obtained from problem $b$ qualify as a solution for problem $a$.

\section{$5 \quad$ Experimental Results}

In earlier sections, we have established the basis of horizontal collision correlation attack along with the strategies to thwart this attack methodology. It is evident from [3] and our previous discussions that ECC scalar multiplication in both Edwards curve and NIST curve is vulnerable to HCCA. Specifically, the Edwards curve implementation incorporating unified formula is extremely vulnerable to HCCA as there exists a pair of multiplication which shares both the operand during execution of point doubling. Hence an adversary is expected to observe sufficiently high similarities when he/she compares the power trace of aforementioned multiplications, sharing both the operands.

We have considered Pearson Correlation metric for our experimental validation which has been extensively recommended in the literature. We show our theory of HCCA protection is practically valid using this metric.We have used SASEBO-GII as the hardware platform for evaluating HCCA and countermeasure. All the algorithms are implemented on cryptographic FPGA of SASEBO-GII (XC5VLX50).

We show results on EM traces of actual ECC scalar multiplication for an underlying Edwards curve. We have implemented Curve1174 on SASEBO-GII evaluation board and have collected around $600 \mathrm{EM}$ traces of scalar multiplication. As we have already mentioned in the previous sections that in Edwards curve unified formula, point doubling involves a pair of field multiplication having both of their operands shared whereas point addition does not have any pair of field multiplications which share both of the operand. Success of HCCA depends upon whether an adversary can distinguish between a pair of field multiplications having both of their operand shared and a pair of field multiplications having no common operand. If the adversary can achieve this, he can distinguish between point doubling and point addition operations which will directly give him the knowledge about secret scalar value. By using the countermeasure, we aim to remove the threat of HCCA. The objective is to make the job of distinguishability between pair of multiplications having no operand shared and pair of multiplications having both of their operand shared difficult. In figure 4 (a), red plot denotes the pair of multiplication with sharing of operands (within doubling) while the blue plot denotes multiplication pair with no sharing (within addition). It demonstrates HCCA attack, when the red plot has a higher correlation value than the blue plot with a success rate of $93.33 \%$ for 600 single scalar multiplication runs. While Figure 4(b) contains a green plot for the pair of multiplications with operand sharing (within the doubling operation), where the operands have been swapped 


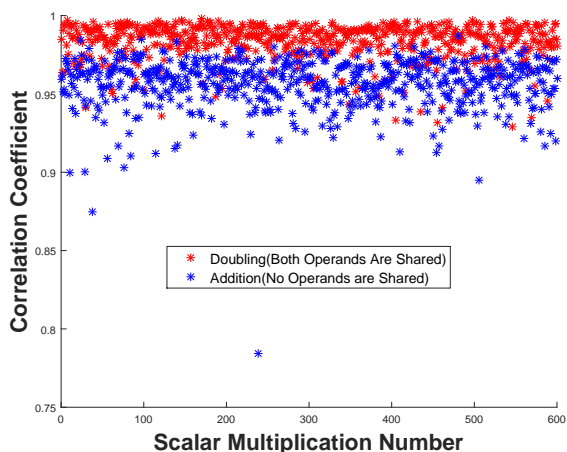

(a) Evaluation of HCCA on Edwards Curve Scalar Multiplier with Correlation

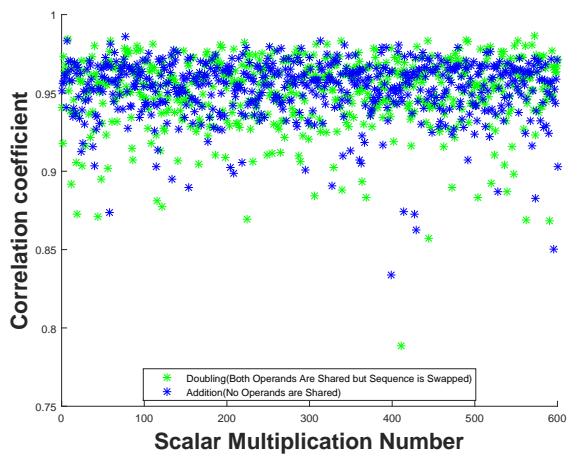

(b) Evaluation of our countermeasure on Edwards Curve Scalar Multiplier with Correlation

Figure 4: Evaluation of HCCA and our countermeasure on Edwards Curve Scalar Multiplier with Correlation: reduces HCCA vulnerability from $93.33 \%$ to $47.5 \%$

as a measure of the countermeasure. Besides, it contains a blue plot which denotes a pair of multiplications with no sharing of operand (within the addition). In this figure, the number of occasions when the green plot (sharing of operands) has a higher correlation than the blue plot (no sharing of operands) is 285 out of 600 cases, which gives HCCA success rate of $47.5 \%$. Thus swapping of operands reduces success rate of HCCA from $93.33 \%$ to $47.5 \%$ as noted using Pearson correlation metric.

\section{Conclusion}

We have shown how the property of asymmetric leakage of field multipliers can be utilized to construct a low-cost countermeasure which is able to defeat the powerful HCCA. We demonstrated how a unified addition (doubling) formula can be converted into a safe sequence where, the information leakage from sharing of operands among field multipliers have been hidden. The process of conversion to the desired safe sequence is achieved through our proposed algorithm (Algorithm 2), once the sequence have been determined through our algorithm there is no runtime overhead requirement for the countermeasure. We have validated HCCA and our proposed countermeasure scheme on a SASEBO platform. This HCCA countermeasure, since zero-cost can be easily integrated with other horizontal attack countermeasures and vertical attack countermeasures involving randomization techniques [11], thus helping in designing a secure ECC-based crypto-module. For an instance, our HCCA countermeasure integrated with a randomization-based countermeasure has been demonstrated in [12], which thwarts a larger class of horizontal attacks.

\section{References}

[1] Frédéric Amiel, Benoit Feix, Michael Tunstall, Claire Whelan, and William P. Marnane. Distinguishing multiplications from squaring operations. In Selected Areas in Cryptography, 15th International Workshop, SAC 2008, Sackville, New Brunswick, Canada, August 14-15, Revised Selected Papers, pages 346-360, 2008. 
[2] Lejla Batina, Lukasz Chmielewski, Louiza Papachristodoulou, Peter Schwabe, and Michael Tunstall. Online template attacks. In Progress in Cryptology - INDOCRYPT 2014 - 15th International Conference on Cryptology in India, New Delhi, India, December 14-17, 2014, Proceedings, pages 21-36, 2014.

[3] Aurélie Bauer, Éliane Jaulmes, Emmanuel Prouff, Jean-René Reinhard, and Justine Wild. Horizontal collision correlation attack on elliptic curves - - extended version -. Cryptography and Communications, 7(1):91-119, 2015.

[4] Daniel J. Bernstein, Peter Birkner, Marc Joye, Tanja Lange, and Christiane Peters. Twisted edwards curves. In Progress in Cryptology - AFRICACRYPT 2008, First International Conference on Cryptology in Africa, Casablanca, Morocco, June 11-14, 2008. Proceedings, pages 389-405, 2008.

[5] Daniel J. Bernstein, Peter Birkner, Marc Joye, Tanja Lange, and Christiane Peters. Twisted edwards curves. In Progress in Cryptology - AFRICACRYPT 2008, First International Conference on Cryptology in Africa, Casablanca, Morocco, June 11-14, 2008. Proceedings, pages 389-405, 2008.

[6] Daniel J. Bernstein and Tanja Lange. Faster addition and doubling on elliptic curves. In Advances in Cryptology - ASIACRYPT 2007, 13th International Conference on the Theory and Application of Cryptology and Information Security, Kuching, Malaysia, December 2-6, 2007, Proceedings, pages 29-50, 2007.

[7] Daniel J. Bernstein and Tanja Lange. Safecurves: choosing safe curves for elliptic-curve cryptography, http://safecurves.cr.yp.to/. 2014.

[8] Eric Brier and Marc Joye. Weierstraß elliptic curves and side-channel attacks. In Public Key Cryptography, 5th International Workshop on Practice and Theory in Public Key Cryptosystems, PKC 2002, Paris, France, February 12-14, 2002, Proceedings.

[9] Benoît Chevallier-Mames, Mathieu Ciet, and Marc Joye. Low-cost solutions for preventing simple side-channel analysis: Side-channel atomicity. IEEE Trans. Computers, 53(6):760-768, 2004.

[10] Christophe Clavier, Benoit Feix, Georges Gagnerot, Christophe Giraud, Mylène Roussellet, and Vincent Verneuil. ROSETTA for single trace analysis. In Progress in Cryptology - INDOCRYPT 2012, 13th International Conference on Cryptology in India, Kolkata, India, December 9-12, 2012. Proceedings, pages 140-155, 2012.

[11] Jean-Sébastien Coron. Resistance against differential power analysis for elliptic curve cryptosystems. In Cryptographic Hardware and Embedded Systems, First International Workshop, CHES'99, Worcester, MA, USA, August 12-13, 1999, Proceedings, pages 292-302, 1999.

[12] Poulami Das, Debapriya Basu Roy, and Debdeep Mukhopadhyay. Exploiting the order of multiplier operands: A low cost approach for HCCA resistance. IACR Cryptology ePrint Archive, 2015:925, 2015.

[13] Harold M. Edwards. A normal form for elliptic curves, 2007.

[14] Junfeng Fan and Ingrid Verbauwhede. An updated survey on secure ECC implementations: Attacks, countermeasures and cost. In Cryptography and Security: From Theory to Applications Essays Dedicated to Jean-Jacques Quisquater on the Occasion of His 65th Birthday, pages 265-282, 2012.

[15] Benoit Feix, Mylène Roussellet, and Alexandre Venelli. Side-channel analysis on blinded regular scalar multiplications. In Progress in Cryptology - INDOCRYPT 2014 - 15th International Conference on Cryptology in India, New Delhi, India, December 14-17, 2014, Proceedings, pages 3-20, 2014.

[16] Daniel Genkin, Adi Shamir, and Eran Tromer. RSA key extraction via low-bandwidth acoustic cryptanalysis. In Advances in Cryptology - CRYPTO 2014 - 34th Annual Cryptology Conference, Santa Barbara, CA, USA, August 17-21, 2014, Proceedings, Part I, pages 444-461, 2014.

[17] Louis Goubin. A refined power-analysis attack on elliptic curve cryptosystems. In Public Key Cryptography - PKC 2003, 6th International Workshop on Theory and Practice in Public Key 
Cryptography, Miami, FL, USA, January 6-8, 2003, Proceedings, pages 199-210, 2003.

[18] Darrel Hankerson, Alfred J. Menezes, and Scott Vanstone. Guide to Elliptic Curve Cryptography. Springer-Verlag New York, Inc., Secaucus, NJ, USA, 2003.

[19] Neil Hanley, HeeSeok Kim, and Michael Tunstall. Exploiting collisions in addition chain-based exponentiation algorithms using a single trace. In Topics in Cryptology - CT-RSA 2015, The Cryptographer's Track at the RSA Conference 2015, San Francisco, CA, USA, April 20-24, 2015. Proceedings, pages 431-448, 2015.

[20] Hüseyin Hisil, Kenneth Koon-Ho Wong, Gary Carter, and Ed Dawson. Twisted edwards curves revisited. In Advances in Cryptology - ASIACRYPT 2008, 14th International Conference on the Theory and Application of Cryptology and Information Security, Melbourne, Australia, December 7-11, 2008. Proceedings, pages 326-343, 2008.

[21] Kouichi Itoh, Tetsuya Izu, and Masahiko Takenaka. Address-bit differential power analysis of cryptographic schemes OK-ECDH and OK-ECDSA. In Cryptographic Hardware and Embedded Systems - CHES 2002, 4th International Workshop, Redwood Shores, CA, USA, August 13-15, 2002, Revised Papers, pages 129-143, 2002.

[22] Kouichi Itoh, Tetsuya Izu, and Masahiko Takenaka. A practical countermeasure against address-bit differential power analysis. In Cryptographic Hardware and Embedded Systems - CHES 2003, 5th International Workshop, Cologne, Germany, September 8-10, 2003, Proceedings, pages 382-396, 2003.

[23] Marc Joye. Highly regular right-to-left algorithms for scalar multiplication. In Cryptographic Hardware and Embedded Systems - CHES 2007, 9th International Workshop, Vienna, Austria, September 10-13, 2007, Proceedings, pages 135-147, 2007.

[24] Marc Joye and Sung-Ming Yen. The montgomery powering ladder. In Cryptographic Hardware and Embedded Systems - CHES 2002, 4th International Workshop, Redwood Shores, CA, USA, August 13-15, 2002, Revised Papers, pages 291-302, 2002.

[25] Kwang Ho Kim, Chol Ok Lee, and Christophe Nègre. Binary edwards curves revisited. In Progress in Cryptology - INDOCRYPT 2014 - 15th International Conference on Cryptology in India, New Delhi, India, December 14-17, 2014, Proceedings, pages 393-408, 2014.

[26] Paul C. Kocher, Joshua Jaffe, and Benjamin Jun. Differential power analysis. In Advances in Cryptology - CRYPTO '99, 19th Annual International Cryptology Conference, Santa Barbara, California, USA, August 15-19, 1999, Proceedings, pages 388-397, 1999.

[27] Patrick Longa. Accelerating the scalar multiplication on elliptic curve cryptosystems over prime fields. IACR Cryptology ePrint Archive, 2008:100, 2008.

[28] Kai Schramm, Thomas J. Wollinger, and Christof Paar. A new class of collision attacks and its application to DES. In Fast Software Encryption, 10th International Workshop, FSE 2003, Lund, Sweden, February 24-26, 2003, Revised Papers, pages 206-222, 2003.

[29] Takeshi Sugawara, Daisuke Suzuki, and Minoru Saeki. Two operands of multipliers in side-channel attack. IACR Cryptology ePrint Archive, 2015:291, 2015.

[30] Colin D. Walter. Sliding windows succumbs to big mac attack. In Cryptographic Hardware and Embedded Systems - CHES 2001, Third International Workshop, Paris, France, May 14-16, 2001, Proceedings, number Generators, pages 286-299, 2001. 


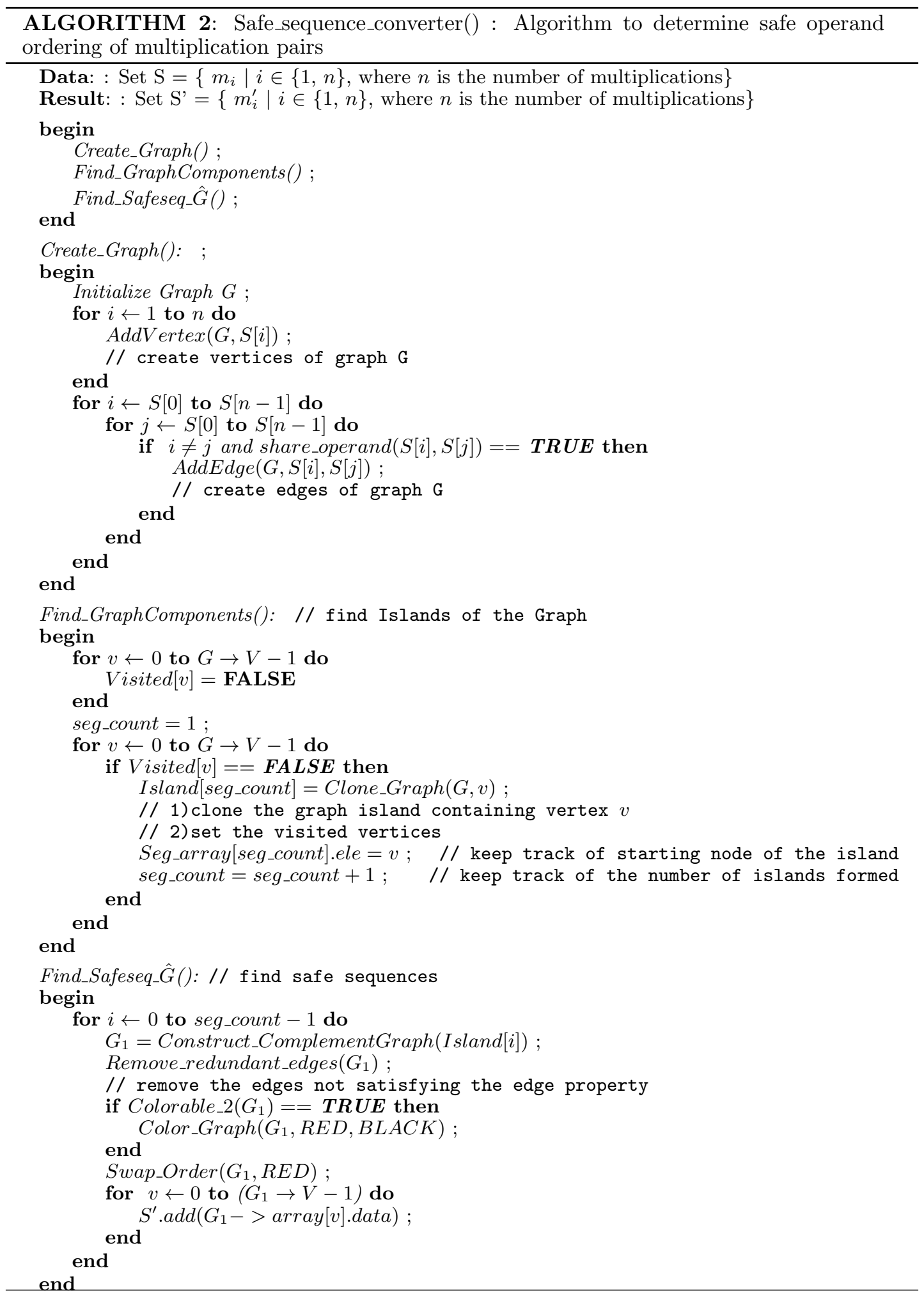

